\title{
New taxa and nomenclatorial acts in volume 164
}

\section{Coleoptera}

\section{Anthicidae}

Anthicus lobanovi Telnov....

Nitorus uyghur Telnov....

Stricticomus kucha Telnov....

\section{Hydraenidae}

Hydraena angelitofelipepunoi Freitag \& Hornyák.. 10 Hydraena buschietanni Freitag \& Hornyák

Hydraena perpusilla Freitag \& Hornyák

\section{Diptera}

\section{Chyromyidae}

Aphaniosoma cypriense Ebejer.

Aphaniosoma pteropus Ebejer.

\section{Carnidae}

Meoneura ibizana Ebejer.

Meoneura micropygialis Ebejer

\section{Heteroptera}

Veliidae

Shaverdinia Zettel \& Laciny

Shaverdinia balkei Zettel \& Laciny

112

Shaverdinia shaverdella Zettel \& Laciny

\section{Lepidoptera}

\section{Lecithoceridae}

Thubana bhutanica Park \& Gielis ........................ 39

Torodora namgaydemae Park \& Gielis.................... 31

\section{Plecoptera}

\section{Perlidae}

Neoperla pangantihoni Pelingen \& Freitag. 